\title{
Identification of the PEA Hysteresis Property Using a Minimum Variance Scheme
}

\author{
Aissa Rebai, Kamel Guesmi, and Boualem Hemici, Member, IACSIT
}

\begin{abstract}
The minimum variance principle is, generally, used for controller's synthesis. In this paper, we propose another use of this principle to identify the hysteresis property of piezoelectric actuators using the Extended Least Squares identification technique adapted for the ARMAX model.
\end{abstract}

IndexTerms - Piezoelectric actuator, nonlinearity, hysteresis, identification.

\section{INTRODUCTION}

The piezoelectric actuators (PEA) based on the inverse piezoelectric effect are used in many fields due to their properties. Indeed, for example they are very used in the ultra-precision applications [1]-[3]. However, the hysteresis property, existing in piezoelectric materials, makes the modeling and the control of PEA difficult. Many nonlinear models was developed in the literature to describe the hysteresis property of piezoelectric actuators such as the Preisach model and its modifications [4]-[8], the Duhem model [9], [10], the Maxwell Resistance Capacitor (MRC) model [11], the Bouc-Wen model [12]-[15], the PrandtlIshlinskii model [16]-[20] and the modified Rayleigh model [21]. A survey on these models can be found in [22]. Furthermore, the experimentation showed that the hysteresisnon-linearity in PEA is not symmetric and many models was proposed in [23]-[25] to describe the asymmetric hysteresis existing in PEA. To compensate the hysteresis behavior of PEA, many intelligent techniques was used such as fuzzy logic [26], [27], neural networks [28], [29], adaptive filter [30], [31], hybrid models [32], NARMAX models [33], [34] and iterative learning control [35]. The most previous models are nonlinear and difficult to implement in on-line which makes the controller synthesis and analysis difficult. To deal with this problem, the PEA can be described by linear models using identification algorithms [36], [37]. In this paper, we propose a technique for the description of the hysteresis property. This technique is based on the modification of the minimum variance controller algorithm to be used for identification purpose. This paper is organized as follows: the extended least squares recursive identification method is described in Section II, then, the proposed minimum variance identification scheme is presented in Section III and before concluding, the proposed approach is validated through simulation results.

Manuscript received March 9, 2014; revised June 20, 2014.

A. Rebai and B. Hemici are with the National Polytechnic School of Algiers, Algeria (e-mail: aissa.rebai@g.enp.edu.dz, bhemici@yahoo.fr).

K. Guesmi is with the CReSTIC, Reims University, France (e-mail: guesmi01@univ-reims.fr).

\section{THE EXTENDED LEAST SQUARES (ELS) IDENTIFICATION METHODS}

The piezoelectric actuator can be described by the ARMAX model of the following expression:

$$
A\left(q^{-1}\right) y(t)=q^{-d} B\left(q^{-1}\right) u(t)+C\left(q^{-1}\right) e(t)
$$

with

$$
\begin{gathered}
A\left(q^{-1}\right)=1+\sum_{i=1}^{n_{A}} a_{i} q^{-i} \\
B\left(q^{-1}\right)=\sum_{i=1}^{n_{B}} b_{i} q^{-i} \\
C\left(q^{-1}\right)=1+\sum_{i=1}^{n_{C}} c_{i} q^{-i}
\end{gathered}
$$

And $y(t), u(t)$ are respectively the output and the input signals, $e(t)$ is a white noise with zero mean value and constant variance and $d$ is pure time delay.

Hence, model (1) can be written as:

$$
\begin{gathered}
y(t+1)=-\sum_{i=1}^{n_{A}} a_{i} y(t+1-i)+\sum_{i=1}^{n_{B}} b_{i} u(t+1-d-i) \\
+e(t+1)+\sum_{i=1}^{n_{C}} c_{i} e(t+1-i)=\theta^{T} \varphi(t)+e(t+1) \\
\theta^{T}=\left[a_{1}, \ldots, a_{n_{A}}, b_{1}, \ldots, b_{n_{B}}, c_{1}, \ldots, c_{n_{C}}\right] \\
\varphi^{T}=\left[-y(t), \ldots,-y\left(t+1-n_{A}\right), u(t-d), \ldots, u\left(t+1-d-n_{B}\right),\right. \\
\left.e(t), \ldots, e\left(t+1-n_{C}\right)\right]
\end{gathered}
$$

If we assume that the estimate of $\theta$ is $\theta$, the estimated output $y$ is given by:

$$
y(t+1)=\theta^{T}(t) \varphi(t)
$$

where $\theta^{T}=\left[a_{1}, \ldots, a_{n_{A}}, \hat{b}_{1}, \ldots, \hat{b}_{n_{B}}, \hat{c}_{1}, \ldots, \hat{c}_{n_{C}}\right]$

The prediction error between the real and the estimated output $\varepsilon(t)$ is defined by:

$$
\varepsilon(t)=y(t+1)-y(t+1)=y(t+1)-\theta^{T}(t) \varphi(t)
$$

We define also the criterion $J(t)$ :

$$
\min _{\theta(t)} J(t)=\frac{1}{2} \sum_{i=1}^{t}\left[y(i)-\theta^{T}(t) \varphi(i-1)\right]^{2}
$$


Minimizing this criterion means:

$$
\frac{\partial J(t)}{\partial \theta(t)}=\sum_{i=1}^{t}\left(-\varphi(i-1)\left[y(i)-\theta^{T}(t) \varphi(i-1)\right]\right)^{T}=0
$$

From (6), the estimated parameters vector $\hat{\theta}$ is obtained as follows:

$$
\theta(t)=\left(\sum_{i=1}^{t} \varphi(i-1) \varphi^{T}(i-1)\right)^{-1}\left[\sum_{i=1}^{t} y(i) \varphi(i-1)\right]
$$

We put:

$$
\theta(t)=F(t) \sum_{i=1}^{t} y(i) \varphi(i-1)
$$

With

$$
F^{-1}(t)=\sum_{i=1}^{t} \varphi(i-1) \varphi^{T}(i-1)
$$

From (9):

$$
\begin{gathered}
F^{-1}(t)=\sum_{i=1}^{t+1} \varphi(i-1) \varphi^{T}(i-1)=\sum_{i=1}^{t} \varphi(i-1) \varphi^{T}(i-1)+\varphi(t) \varphi^{T}(t) \\
=F^{-1}(t)+\varphi(t) \varphi^{T}(t)
\end{gathered}
$$

Thus, the adaptation gain $F(t+1)$ is given by:

$$
F(t+1)=F(t)-\frac{F(t) \varphi(t) \varphi^{T}(t) F(t)}{1+\varphi^{T}(t) F(t) \varphi(t)}
$$

From (8):

$$
\sum_{i=1}^{t} y(i) \varphi(i-1)=F^{-1}(t) \theta(t)
$$

For $i=1, \ldots ., t+1$, we have:

$$
\begin{gathered}
\sum_{i=1}^{t+1} y(i) \varphi(i-1)=\sum_{i=1}^{t} y(i) \varphi(i-1)+y(t+1) \varphi(t) \\
=F^{-1}(t) \theta(t)+y(t+1) \varphi(t)
\end{gathered}
$$

From (11) and (12) we have:

$$
\begin{gathered}
F^{-1}(t+1) \theta(t+1)=F^{-1}(t) \theta(t)+y(t+1) \varphi(t) \\
=F^{-1}(t) \theta(t+1)+\varphi(t) \varepsilon(t+1)
\end{gathered}
$$

We obtain:

$$
\theta(t+1)=\theta(t)+F(t+1) \varphi(t) \varepsilon(t+1)
$$

where

$$
F(t+1)=F(t)-\frac{F(t) \varphi(t) \varphi^{T}(t) F(t)}{1+\varphi^{T}(t) F(t) \varphi(t)}
$$

And

$\theta(t)$ : Estimated parameters vector

$F(t)$ : Adaptation gain

$\varepsilon(t)$ : Prediction error

$\varphi(t)$ : Observations vector

Equations (13) and (14) are called parametric adaptation algorithm (PAA) which is used for all recursive identification techniques. To validate the identified model, the estimations of the normalized autocorrelations $R N(i)$ are calculated as follows:

$$
R N(i)=\frac{R(i)}{R(0)} ; i=1, \ldots, \max \left(n_{A}, n_{B}+d\right)
$$

With

$$
R(i)=\frac{1}{N} \sum_{t=1}^{N} \varepsilon(t) \varepsilon(t-i) ; R(0)=\frac{1}{N} \sum_{t=1}^{N} \varepsilon^{2}(t)
$$

The identified model is valid if $|R N(i)|<0.15$ [38].

\section{DESCRIPTION OF THE MINIMUM VARIANCE IDENTIFICATION SCHEME}

In this paper we modified the minimum variance controller scheme to be used for identification as shown in Fig. 1. Indeed, instead of using as input a reference signal, we use the estimated output. In the diagram of Fig. 1 to be used for identification purpose, $y(t)$ is the system output, $u(t)$ is the control signal and $y(t+1+d)$ is the predicted output. $R$ and $S$ are the regulator polynomials and are given by [39], [40]:

$$
\begin{gathered}
R\left(q^{-1}\right)=\sum_{i=1}^{n_{R}} r_{i} q^{-i} \\
S\left(q^{-1}\right)=\sum_{i=1}^{n_{S}} S_{i} q^{-i}
\end{gathered}
$$

where $n_{R}=n_{B}+d+1$

$$
n_{S}=n_{A}-1
$$

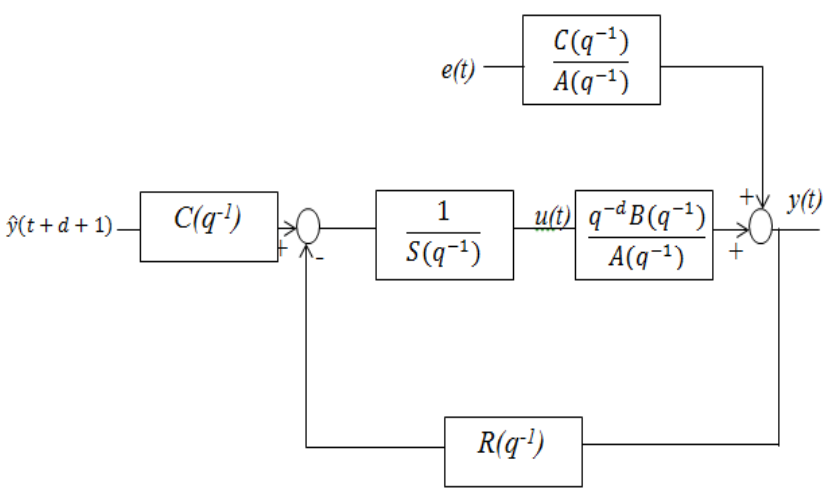

Fig. 1. Proposed minimum variance identification scheme.

From the diagram we have:

$$
C\left(q^{-1}\right) y(t+d+1)=R\left(q^{-1}\right) y(t)+S\left(q^{-1}\right) u(t)
$$


For the case $d=0$, we have

$$
\begin{gathered}
y(t+1)=-\sum_{i=1}^{n_{C}} c_{i} y(t+1-i)+R\left(q^{-1}\right) y(t)+S\left(q^{-1}\right) u(t) \\
=\theta^{T} \varphi(t)
\end{gathered}
$$

Then, the PAA of the equations (13) and (14) can be applied with

$$
\begin{gathered}
\theta^{T}=\left[\hat{r}_{0}, \ldots, \hat{r}_{n_{R}}, \hat{s}_{0}, \ldots, \hat{s}_{n_{S}}, \hat{c}_{1}, \ldots, \hat{c}_{n_{C}}\right] \\
\varphi^{T}=\left[y(t), \ldots, y\left(t-n_{R}\right), u(t-d), \ldots, u\left(t-n_{S}\right), y(t), \ldots, y\left(t-n_{C}\right)\right]
\end{gathered}
$$

\section{Simulation RESUlts}

In this section, we use the algorithms of equations (13) and (14) with the parameters and observations vectors of the proposed approach to identify the hysteresis property of the piezoelectric actuator.

In order to validate the proposed approach, we use the Matlab environment to implement the different algorithms. The data file (I/O file) used for the identification corresponds to the piezoelectric actuator APA-120ML excited by a sinusoidal signal of frequency $50 \mathrm{~Hz}$ :

$$
u(t)=68.5 \sin \left(2 \_\times 50 t+0.44\right)+61.5(v) .
$$

The piezoelectric actuators are generally modeled by a second order system, therefore the polynomials orders of the model are selected as follows: $n_{A}=2, n_{B}=2, n_{C}=1$ and $d=0$. which corresponds to $n_{R}=1, n_{S}=1$ and $n_{C}=1$.

The identification results and the convergence of the regulator parameters are shown in Fig. 2a, Fig. 2b, and Fig. 2c. According to Fig. 2a, we can remark that the proposed approach can ensure satisfactory results with an identified behavior of PEA close to the original one.

To evaluate proposed approach performances, the identification relative error and the values of its autocorrelation function are given in Fig. 3a and Fig. 3b, respectively. These figures shows that the maximum relative error of identification is $5 \%$, also $|R N(i)|$ are all small than 0.15 . Therefore, the prediction error tends towards a white noise which validates and draws clearly the performance of the proposed approach.

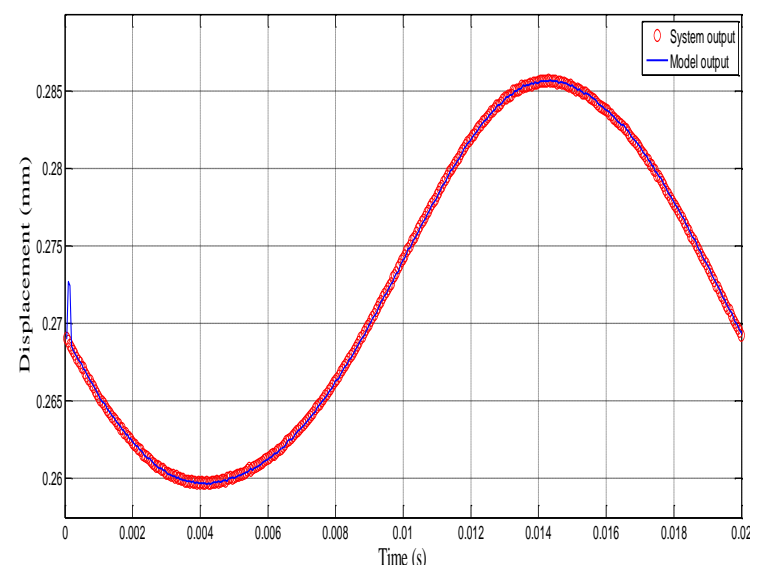

(a) Outputs comparison.

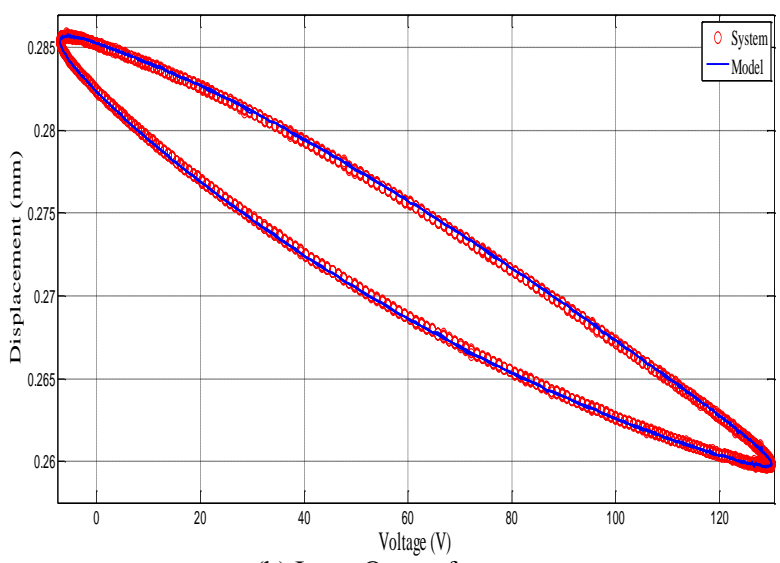

(b) Input-Output feature.

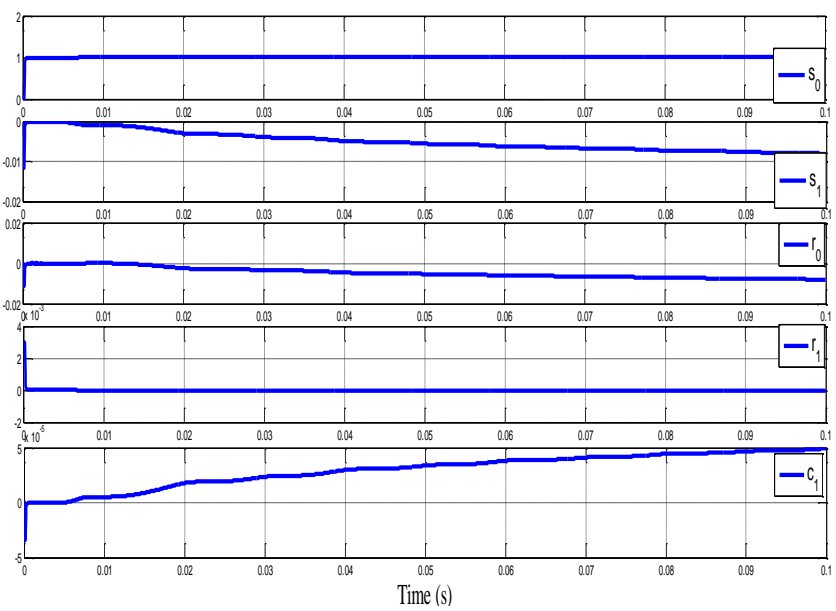

(c) Variation of the regulator parameters.

Fig. 2. Identification results.

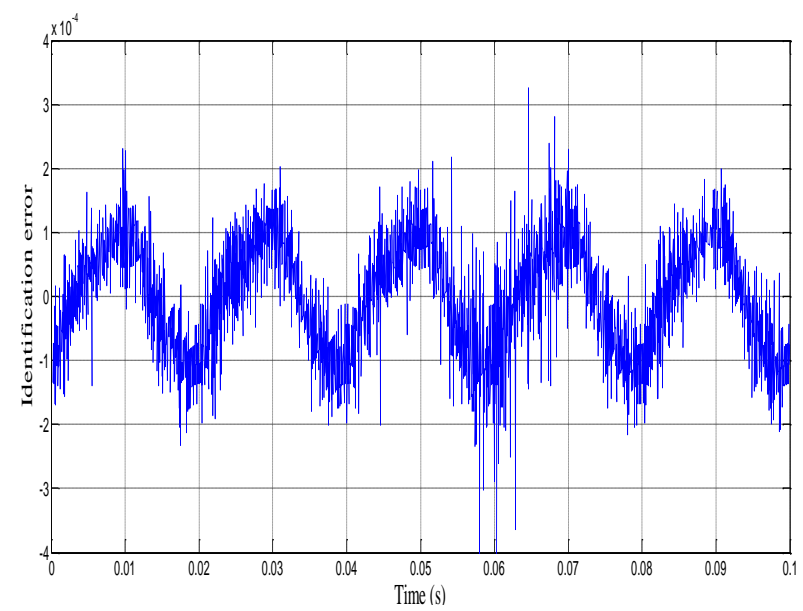

(a) Identification error.

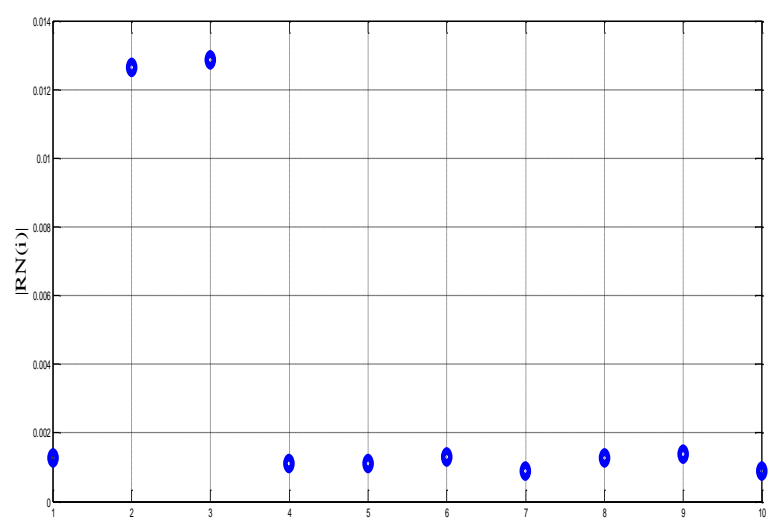

(b) Error autocorrelation.

Fig. 3. Validation of the technique. 


\section{CONCLUSION}

In this paper, an identification scheme was proposed based on the minimum variance principle to identify the hysteresis property existing in PEA. An on-line model is constructed based on the ARMAX model and the extended least squares algorithm is used to represent this phenomenon.

Simulation results validated and showed the performance of the proposed approach. Furthermore, they showed also that the ARMAX model can characterize well the hysteresis non linearity in piezoelectric actuators which facilitates the analysis and the control of these devices.

\section{REFERENCES}

[1] Q. Xu and Y. Li, "Tracking performance characterization and improvement of a piezoactuated micropositioning system based on an empirical index," Robotics and Computer -Integrated Manufacturing, vol. 26, no. 6, pp. 744-752, 2010.

[2] B. Zhang and Z. Zhu, "Developing a linear piezomotor with nanometer resolution and high stiffness," IEEE/ASME Transactions on Mechatronics, vol. 2, no. 1, pp. 22 - 29, 1997.

[3] H. Haitjema, "Dynamic probe calibration in the $1 \mathrm{~m}$ region with nanometer accuracy," Precision Engineering, vol. 19, pp. 98-104, 1996.

[4] F. Preisach, "Über die magnetische nachwirkung," Zeitschrift für Physik A Hadrons and Nuclei, vol. 94, no. 5-6, pp. 277 - 302, 1935.

[5] S. Xiao and Y. Li, "Modeling and high dynamic compensating the rate-dependent hysteresis of piezoelectric actuators via a novel modified inverse Preisach model," IEEE Transactions On Control Systems Technology, pp. 1-9, 2012.

[6] J. Y. Peng and X. B. Chen, "Novel models for one-sided hysteresis of piezoelectric actuators," Mechatronics, vol. 22, pp. 757 - 765, 2012.

[7] H. Hu and R. B. Mrad, "On the classical Preisach model for hysteresis in piezoceramic actuators," Mechatronics, vol. 13, pp. 85-94, 2003.

[8] P. Ge and M. Jouaneh, "Generalized Preisach model for hysteresis nonlinearity of piezoceramic actuators," Precision Engineering, vol 20, pp. $99-111,1997$.

[9] P. Duhem, "Die dauernden aenderungen und die thermodynamik," I. Z Phys. Chem, pp. 543-589, 1897.

[10] C. Hui, T. Yonghong, Z. Xingpeng, D. Ruili, and Z. Yahong, "Identification of dynamic hysteresis based on Duhem model," in Proc. 4th International Conference on Intelligent Computation Technology and Automation, 2011, pp. 810-814.

[11] M. Goldfarb and N. Celanovic, "Modeling piezoelectric stack alctuators for control of micromanipulation," IEEE Control Systems, pp. 69-79, June 1997.

[12] R. Bouc, "Modèle mathématique d'hystérésis," Acoustica, vol. 21, pp. $16-25,1971$

[13] O. Gomis-Bellmunt, F. Ikhouane, and D. Montesinos-Miracle, "Control of a piezoelectric actuator considering hysteresis," Journal of Sound and Vibration, vol. 326, pp. 383 - 399, 2009.

[14] S. Xiao and Y. Li, "Dynamic compensation and $H_{\infty}$ control for piezoelectric actuators based on the inverse Bouc-Wen model," Robotics and Comp.-Integrated Manufac., vol. 30, pp. 47 - 54, 2014.

[15] Z. Y. Wang and J. Q. Mao, "On PSO based Bouc-Wen modeling for piezoelectric actuator," in Proc. ICIRA 2010, pp. 125-134.

[16] I. Mayergoyz, Mathematical Models of Hysteresis and Their Applications, Elsevier, 2003.

[17] H. Janocha and K. Kuhnen, "Real-time compensation of hysteresis and creep in piezoelectric actuators," Sensors Actuators: A Phys, vol. 79 pp. 83-89, 2000.

[18] M. J. Yang, G. Y. Gu, and L. M. Zhu, "Parameter identification of the generalized Prandtl-Ishlinskii model for piezoelectric actuators using modified particle swarm optimization," Sensors and Actuators A: Phys., vol. 189, pp. 254-265, 2013.

[19] V. T. Liu and C. H. Li, "Modeling hysteresis of the piezoactuators with Prandtl-Ishlinskii model," in Proc. the 3rd International Conference on Innovative Computing Information and Control, 2008.

[20] Y. Guo et al., "Modeling of rate-dependent hysteresis for piezoelectric actuator with MPI model-based Hammerstein system," in Proc. ICIRA 2012, pp. 281-290.

[21] J. Park et al., "Hysteresis compensation of piezoelectric actuators: The modified Rayleigh model," Ultrasonics, vol. 50, pp. 335-339, 2010.

[22] A. Rebai et al., "Modeling of the hysteresis property in piezoelectric actuators a survey," in Proc. Int. Conf. on Elect. Eng. and Autom. Contr., Setif -Algeria, November 24-26 2013.
[23] G. Aguirre, T. Janssens, H. V. Brussel, and F. Al-Bender, "Asymmetric hysteresis compensation in piezoelectric actuators," Mechanical Systems and Signal Processing, vol. 30, pp. 218-231, 2012.

[24] W. Zhua and D. H. Wang, "Non-symmetrical Bouc-Wen model for piezoelectric ceramic actuators," Sensors and Actuators A: Physical, vol. 181, pp. 51-60, 2012.

[25] H. Jiang, H. Ji, J. Qiu, and Y. Chen, "A modified Prandtl-Ishlinskii model for modeling asymmetric hysteresis of piezoelectric actuators," IEEE Tran. on Ultrasonics, Ferroelectrics, and Frequency Control, vol. 57, no. 5, pp. 1200-1210, May 2010.

[26] P. Li, F. Yan, C. Ge, X. Wang, L. Xu, J. Guo, and P. Li, “A simple fuzzy system for modelling of both rate-independent and rate-dependent hysteresis in piezoelectric actuators," Mechanical Systems and Signal Processing, vol. 36, pp. 182 - 192, 2013.

[27] P. Li, G. Gu, L. Lai, and L. Zhu, "Hysteresis modeling of piezoelectric actuators using the fuzzy system," in Proc. ICIRA, 2010, pp. 114- 124

[28] X. Zhang, Y. Tan, and M. Su, "Modeling of hysteresis in piezoelectric actuators using neural networks," Mechanical Systems and Signal Processing, vol. 23, pp. 2699 - 2711, 2009

[29] W. Li and X. Chen, "Compensation of hysteresis in piezoelectric actuators without dynamics modeling," Sensors and Actuators A: Physical, vol. 199, pp. 89 - 97, 2013.

[30] J. Minase, T. F. Lu, B. Cazzolato, and S. Grainger, "Adaptive identification of hysteresis and creep in piezoelectric stack actuators," Int. J. Adv. Manuf Technol, vol. 46, pp. 913-921, 2010.

[31] X. Liua, Y. Wanga, J. Genga, and Z. Chen, "Modeling of hysteresis in piezoelectric actuator based on adaptive filter," Sensors and Actuator A: Physical, vol. 189, pp. 420-428, 2013.

[32] Y. T. X. Zhanga, "A hybrid model for rate-dependent hysteresis in piezoelectric actuators," Sensors and Actuators A: Physical, vol. 157, pp. 54-60, 2010.

[33] L. Denga and Y. Tan, "Modeling hysteresis in piezoelectric actuators using NARMAX models," Sensors and Actuators A: Physical, vol. 149, pp 106-112, 2009.

[34] L. Deng and Y. Tan, "Modeling of rate-dependent hysteresis in piezoelectric actuators," in Proc. 17th IEEE International Conference on Control Applications, 2008, pp. 978-982.

[35] L. Liu, K. K. TAN, A. S. Putra, and T. H. Lee, "Compensation of hysteresis in piezoelectric actuator with iterative learning control," $J$ Control Theory Appl, vol. 8, no. 2, pp. 176-180, 2010.

[36] T. Zhang, H. G. Li, and G. P. Cai, "Hysteresis identification and adaptive vibration control for a smart cantilever beam by a piezoelectric actuator," Sensors and Actuators: A Physical, 2013.

[37] B. J. Sung, E. W. Lee, and I. S. Kim, "Displacement control of piezoelectric actuator using the PID controller and system identification method," IEEE Proceedings, 2008.

[38] I. D. Landau and G. Zito, Digital Control Systems: Design, Identification and Implementation, Springer, 2006.

[39] Z. Li and R. J. Evans, "Minimum variance control of linear time-varying systems," Automatica, vol. 33, 1997.

[40] Z. Li et al., "Minimum variance prediction for linear time-varying systems," Automatica, vol. 33, pp. 617-618, 1997.

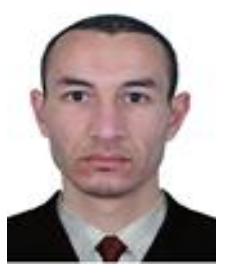

Aissa Rebai received the B.Eng. and master degree in control engineering from National Polytechnic Schoo of Algiers, in 2012. He is currently a PhD student at the Department of Control, National Polytechnic School of Algiers, Algeria.

His research interests include systems identification, intelligent control of mechatronic systems and optimization algorithms.

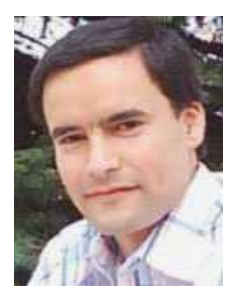

Kamel Guesmi received the BEng degree in electrical engineering from the University of Djelfa, Algeria, in 2000, the MSc and $\mathrm{PhD}$ degrees in electrical engineering from Reims University, France, in 2003 and 2006, respectively. Currently, he is an associate professor in the Department of Electrical Engineering, Djelfa University, Algeria.

His research interests include intelligent control, robust control, power electronics and nonlinear

dynamics

Boualem Hemici is currently an associate professor at National Polytechnic School of Algiers, Algeria.

His research interests include robotics, robust control and technology of renewable energy. 